\title{
Analyst
}

CORRECTION

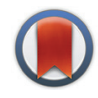

CrossMark

Cite this: Analyst, 2016, 141, 5659

DOI: $10.1039 / c 6 a n 90078$

www.rsc.org/analyst

\section{Correction: Resonance Raman spectroscopy as an in situ probe for monitoring catalytic events in a Ru-porphyrin mediated amination reaction}

\author{
Paolo Zardi, ${ }^{a}$ Emma Gallo, ${ }^{a}$ Gregory A. Solan ${ }^{\mathrm{b}}$ and Andrew J. Hudson* ${ }^{\mathrm{b}}$
}

Correction for 'Resonance Raman spectroscopy as an in situ probe for monitoring catalytic events in a Ru-porphyrin mediated amination reaction' by Paolo Zardi et al., Analyst, 2016, 141, 3050-3058.

The authors regret that Emma Gallo was incorrectly affiliated with ${ }^{a}$ Department of Chemical Sciences, University of Padova, Via Marzolo 1, 35131 Padova, Italy; the correct affiliation for Professor Gallo is Chemistry Department, Università degli Studi di Milano, Via Golgi 19, 20136 Milan, Italy. E-mail: emma.gallo@unimi.it.

The Royal Society of Chemistry apologises for these errors and any consequent inconvenience to authors and readers.

${ }^{a}$ Department of Chemical Sciences, University of Padova, Via Marzolo 1, 35131 Padova, Italy

${ }^{b}$ Department of Chemistry, University of Leicester, Leicester, LE1 7RH, UK. E-mail: andrew.hudson@leicester.ac.uk 